\title{
Identification of a Five-Gene Signature for Predicting Survival in Malignant Pleural Mesothelioma Patients
}

\author{
Yiyang Bai't, Xiao Wang ${ }^{1+}$, Jia Hou' ${ }^{1}$, Luying Geng', Xuan Liang ${ }^{1}$, Zhiping Ruan', \\ Hui Guo ${ }^{1}$, Kejun Nan ${ }^{1,2 *}$ and Lili Jiang ${ }^{1 *}$ \\ ${ }^{1}$ Department of Medical Oncology, The First Affiliated Hospital of Xi'an Jiaotong University, Xi'an, China, ${ }^{2}$ Oncology Hospital, \\ Xi'an International Medical Center Hospital, Xi'an, China
}

OPEN ACCESS

Edited by:

Madhuchhanda Bhattacharjee,

University of Hyderabad, India

Reviewed by:

Hemant Ritturaj Kushwaha,

Jawaharlal Nehru University, India

Mikael Boden,

The University of Queensland,

Australia

*Correspondence:

Kejun Nan

nankj@163.com

Lili Jiang

jiangli8499@163.com

${ }^{\dagger}$ These authors have contributed equally to this work

Specialty section:

This article was submitted to

Computational Genomics,

a section of the journal

Frontiers in Genetics

Received: 31 March 2020

Accepted: 21 July 2020

Published: 07 August 2020

Citation:

Bai Y, Wang X, Hou J, Geng L,

Liang X, Ruan Z, Guo H, Nan K and

Jiang $L$ (2020) Identification of a

Five-Gene Signature for Predicting

Survival in Malignant Pleural

Mesothelioma Patients.

Front. Genet. 11:899.

doi: 10.3389/fgene.2020.00899
Malignant pleural mesothelioma (MPM), predominantly caused by asbestos exposure, is a highly aggressive cancer with poor prognosis. The staging systems currently used in clinics is inadequate in evaluating the prognosis of MPM. In this study, a five-gene signature was developed and enrolled into a prognostic risk score model by LASSO Cox regression analysis based on two expression profiling datasets (GSE2549 and GSE51024) from Gene Expression Omnibus (GEO). The five-gene signature was further validated using the Cancer Genome Atlas (TCGA) MPM dataset. Univariate and multivariate Cox analyses proved that the five-gene signature was an independent prognostic factor for MPM. The signature remained statistically significant upon stratification by Brigham stage, AJCC stage, gender, tumor size, and lymph node status. Time-dependent receiver operating characteristic (ROC) curve indicated good performance of our model in predicting 1- and 2-years overall survival in MPM patients. The C-index was 0.784 for GSE2549 and 0.753 for the TCGA dataset showing moderate predictive accuracy of our model. Furthermore, Gene Set Enrichment Analysis suggested that the five-gene signature was related to pathways resulting in MPM tumor progression. Together, we have established a five-gene signature significantly associated with prognosis in MPM patients. Hence, the five-genes signature may serve as a potentially useful prognostic tool for MPM patients.

Keywords: malignant pleural mesothelioma, gene expression, signature, overall survival, prognosis

\section{INTRODUCTION}

Malignant pleural mesothelioma (MPM), the most common form of malignant mesothelioma, is a highly aggressive neoplasm arising from the pleural mesothelial tissues covering the lung and is predominantly associated with occupational and environmental exposure to asbestos fibers (Wagner et al., 1960; Walker et al., 1983). Centers for Disease Control and Prevention (CDC) reports the annual number of malignant mesothelioma deaths increased by $4.8 \%$, from 2479 in

Abbreviations: AJCC, American Joint Commission on Cancer; AUC, area under curve; CI, confidence interval; DEGs, differentially expressed genes; GEO, Gene Expression Omnibus; GSEA, Gene Set Enrichment Analysis; HR, hazard ratio; IMIG, International Mesothelioma Interest Group; MPM, malignant pleural mesothelioma; OS, overall survival; ROC, receiver operating characteristic curve; SMRP, soluble mesothelin-related protein; TCGA, the Cancer Genome Atlas; UICC, Union for International Cancer Control. 
1999 to 2579 in 2015, in the United States (Mazurek et al., 2017). Though it is still considered a rare disease, as many as 3000 new cases are diagnosed annually in United States alone (Vogelzang et al., 2003). Considering the latency period between the first asbestos exposure to MPM development often ranges anywhere between 20 and 71 years, the global incidence of MPM will likely be on the rise (Lin et al., 2019). Although the time from exposure to onset is long, the progression from onset is rapid. MPM patients often have non-specific symptoms at first which makes diagnosis extremely challenging at early stage. Moreover, the lack of an accurate and universally accepted staging system makes it even harder for investigation and treatment of the disease, leading to the poor prognosis of MPM (Rusch, 1995; Kindler et al., 2018).

Researchers have found several clinicopathological factors associated with poor prognosis of MPM, such as the male gender, elevated serum lactate dehydrogenase levels, chest pain, thrombocytosis, non-epithelial histology, and age $>75$ years (Curran et al., 1998; Herndon et al., 1998). Simultaneously, potent biomarkers have been studied in relation to pathogenesis, diagnosis and prognosis of MPM. To date, non-tissuebased biomarkers have been characterized, including soluble mesothelin-related protein (SMRP), osteopontin and fibulin-3 (Hollevoet et al., 2012; Kindler et al., 2018). However, none of these biomarkers being evaluated at this time for MPM have demonstrated sufficiently rigorous prospective or blinded validation to recommend their use (Kindler et al., 2018). Some studies have evaluated the diagnostic value of microRNA to differentiate MPM from normal pleural mesothelial proliferation or other carcinomas (Busacca et al., 2010; Benjamin et al., 2010; Balatti et al., 2011). The six-microRNA signature constructed by Kirschner et al. (2015) was reported to predict the survival of MPM patients. However only a small number of long and short-term survivors were compared following extrapleural pneumonectomy, and normal samples were not included as a control, thereby limiting the use of this specific signature (Kirschner et al., 2015). Furthermore, an increasing number of studies using gene expression profiling have primarily discovered new oncogenes or tumor suppressor genes or have simply used MPM tumor samples without normal samples to construct the prognostic model. For instance, Gordon et al. developed and validated a nine-ratio (six gene) signature to differentially diagnose MPM from adenocarcinoma, and they also defined a molecular classification of MPM using transcriptional profiling by microarray, however did not independently validate the results (Gordon et al., 2002, 2005). The study took inspiration from previous research and concerning the difficulty to apply the MPM staging system into clinical work to make accurate evaluation of prognosis for MPM patients (Rusch et al., 2012), it is of great value to use bioinformatics methods to discover prognostic genes between MPM tumor and normal tissues as possible biomarkers and construct a risk-score model to clarify MPM patients into high- and low-risk subgroups, leveraging an objective approach in MPM patients' prognosis evaluation.

In this study, based on the gene expression profiling data from GEO and TCGA dataset, we developed and validated a reliable five-gene signature model independent of clinicopathological factors that improved the risk stratification for MPM patients.

\section{MATERIALS AND METHODS}

\section{Datasets}

The two gene expression arrays of human MPM datasets GSE2549 (Gavin et al., 2005) and GSE51024 (Suraokar et al., 2014a) were derived from the Gene Expression Omnibus $(\mathrm{GEO})^{1}$, which is a database repository of high throughput gene expression data and hybridization arrays, chips, microarrays. GSE2549 and GSE51024 sets were conducted on GPL96 (Affymetrix Human Genome U133A Array) and GPL570 (Affymetrix Human Genome U133 Plus 2.0 Array) platforms respectively. The GSE2549 dataset includes 40 discarded human MPM tumor specimens and 9 normal specimens in which four are normal lung specimens and five are normal pleura. The GSE51024 dataset includes 41 MPM tumor tissues along with 41 paired normal tissues. For the validation of TCGA set, we downloaded the mRNA expression data (RNA-seq in FPKM value) which includes 85 MPM patients together with the clinical information from the Cancer Genome Atlas².

\section{Differential mRNA Analysis}

To identify the differentially expressed genes (DEGs), GSE2549 and GSE51024 MPM datasets were employed. After annotating the probes into gene symbols by the platform annotation files, we used R package LIMMA (Ritchie et al., 2015) to get the DEGs between the tumor and normal tissue following the criteria of adjusted $P$-value $<0.05, \mid \log _{2}$ FoldChange $>1$ in both sets. Finally, by overlapping the upregulated and downregulated DEGs from the two datasets, we got the DEGs in both datasets.

\section{Construction of the Prognostic Model by LASSO Cox Regression}

By performing the univariate Cox regression analysis on the candidate DEGs from the two discovery sets, we calculated the correlation between each gene and the overall survival time of each patient and investigated the genes having strongest association with the patients' overall survival time following the criteria of $P<0.05$. R package "survival" was used to perform the univariate Cox regression analysis (Gordon et al., 2020; Terry and Therneau, 2020). The least absolute shrinkage and selection operator (LASSO) Cox regression with 10-time cross validation was used to choose the penalty regularization parameter $\lambda$ (Gui and Li, 2005). The coefficient of each gene was forced to shrink to zero which eliminated the correlation between the selected genes and prevented the model from being overfitting. By applying the minimum deviance, lamda.min, genes were selected. $\mathrm{R}$ package "glmnet" was used to perform LASSO Cox regression analysis (Goeman, 2010; Friedman et al., 2020). Together with the coefficient of each gene generated by multivariate Cox regression analysis, the prognostic risk score model was constructed. $\mathrm{R}$ package "survminer" was used to perform the multivariate Cox regression analysis (Kassambara et al., 2020). Based on the expression of each gene discovered, each patient's risk score was

\footnotetext{
${ }^{1}$ https://www.ncbi.nlm.nih.gov/geo

${ }^{2}$ https://www.cancer.gov/about-nci/organization/ccg/research/structuralgenomics/tcga
} 
calculated according to the risk score model. The risk score model was then used to evaluate the prognosis of MPM patients.

$$
\text { Risk score }=\sum_{i=1}^{n} \text { Coeffient } i^{*} \text { Expression of Gene } i
$$

\section{Construction of the Prognostic Model by Three Other Methods}

To validate the prognostic model constructed by LASSO Cox regression method. Forward stepwise regression, bidirectional stepwise regression and relaxed LASSO methods were introduced to rebuild the model. Prognosis related genes detected by univariate Cox regression analysis were used to perform the analyses for the three methods. In forward stepwise regression analysis, genes were ranked by their z-score, which represented their predictive power. The gene with the highest $z$-score was first enrolled and gene was subsequently added according to the z-score rank list from high to low. The AIC (Akaike information criterion) for each model was calculated new gene was terminated being added to the model if the AIC stopped decreasing which indicated the genes already enrolled giving the best prognostic performance. The bidirectional stepwise regression procedure included the iterations between the "forward" and "backward" steps. R package "My.stepwise.coxph" was used to do the analysis (My.stepwise.coxph, 2017). We found the R package "fastcox" was for Lasso and Elastic-Net penalized Cox's regression in high dimensions models and it could help realize the relaxed lasso analysis (Yang and Zou, 2013a,b). The penalty regularization parameter $\lambda$ was chosen via 10-time cross-validation. By applying the minimum deviance, lamda.min, genes were selected. The genes selected by each of the three methods were then enrolled in the multivariate Cox regression analysis respectively and the risk score models were then established with the method mentioned above.

\section{Survival Analysis and ROC Analysis}

According to the risk score formula, we calculated the risk score for each patient in GSE2549 discovery set and TCGA validation set. Patients from the two datasets were then divided into lowrisk and high-risk groups respectively with the median cutoff of the risk score. Kaplan-Meier survival curves were performed to evaluate whether there was significant difference between the low-risk and high-risk groups by log-rank test with $P<0.05$. Univariate and multivariate analyses were conducted to see whether the five-gene signature could be a prognostic factor for MPM independent of other clinicopathological factors. $P<0.05$ was considered significant. Hazard ratios and $95 \%$ confidence interval were also calculated. Univariate and multivariate analyses were performed using IBM SPSS version 23.0 (IBM Corp., NY, United States). Time-dependent receiver operating characteristic (ROC) curve analyses were conducted to evaluate the prognostic effectiveness of the risk score model compared with other clinicopathological factors and one literature model. We used R package "survivalROC" to perform ROC analysis (Heagerty et al., 2000; Patrick, 2013). C-index was calculated by R package "survival" (Terry and Therneau, 2020). R package
"compareC" was used to perform the comparison of C-index value (Le Le Kang, 2015). The method was for statistical comparison of two diagnostic or predictive systems, of which they could either be two biomarkers or two fixed algorithms, in terms of their $C$ indices (Kang et al., 2015). Z score test was used for hypothesis testing.

\section{Functional Enrichment Analysis}

Gene Set Enrichment Analysis was conducted between the low-risk and high-risk groups to predict the possible molecular mechanisms responsible for the poor prognosis of MPM. Molecular Signatures Database (MSigDB) C2 Canonical pathways gene set database was used to screen the significant pathways with the criteria of $|\mathrm{NES}|$ (normalized enrichment score) $>1$, NOM $P<0.05$ and FDR (false discovery rate) $q<0.05$ after performing 1000 permutations (Liberzon et al., 2011). The enrichment analysis was performed by GSEA $4.0 .3^{3}$.

\section{RESULTS}

\section{Identification of Candidate DEGs}

To obtain the differentially expressed genes (DEGs) between human MPM tumor and normal tissues, two expression datasets GSE2549 and GSE51024 were enrolled as discovery datasets (Figure 1). We firstly screened for the DEGs between MPM tumor and normal tissues in these two datasets using LIMMA analysis in $\mathrm{R}\left(q<0.05, \mid \log _{2}\right.$ Fold Change $\left.\mid>1\right)$. A total of 1438 DEGs, including 837 upregulated genes and 601 downregulated genes, were identified in GSE2549 between 40 MPM tumor specimens and 9 normal specimens. A total of 727 DEGs, including 211 upregulated genes and 516 downregulated genes were screened in GSE51024 between 41 MPM tumor tissues and 41 paired normal tissues. The volcano plots were generated for both datasets (Figure 2A). Moreover, 92 upregulated genes and 133 downregulated genes overlapped between GSE2549 and GSE51024 (Figure 2B). Therefore, a total of 225 candidate DEGs were selected.

\section{Construction and Validation of the Prognostic Risk Score Model for MPM}

By performing univariate Cox regression analysis between these 225 candidate DEGs and survival data of discovery set GSE2549, 36 genes were detected $(P<0.05)$. A LASSO Cox regression analysis together with 10 -time cross validation was then conducted to eliminate the number of genes and select those with non-zero coefficient (Figure 2C). Five genes were identified and using multivariate Cox regression analysis, the coefficient of each gene was calculated. Therefore, a five-gene signature risk score model was developed based on the five genes along with their coefficients and gene expression level. Risk score $=0.1197 \times$ expression of $\mathrm{CDH} 2+0.6824 \times$ expression of CKS $2+0.5594 \times$ expression of KIF11 $+0.7141 \times$ expression of KIF18B $+0.5004 \times$ expression of LOX. To confirm the validity of the signature, we used three other methods,

\footnotetext{
${ }^{3}$ https://www.gsea-msigdb.org/gsea/index.jsp
} 


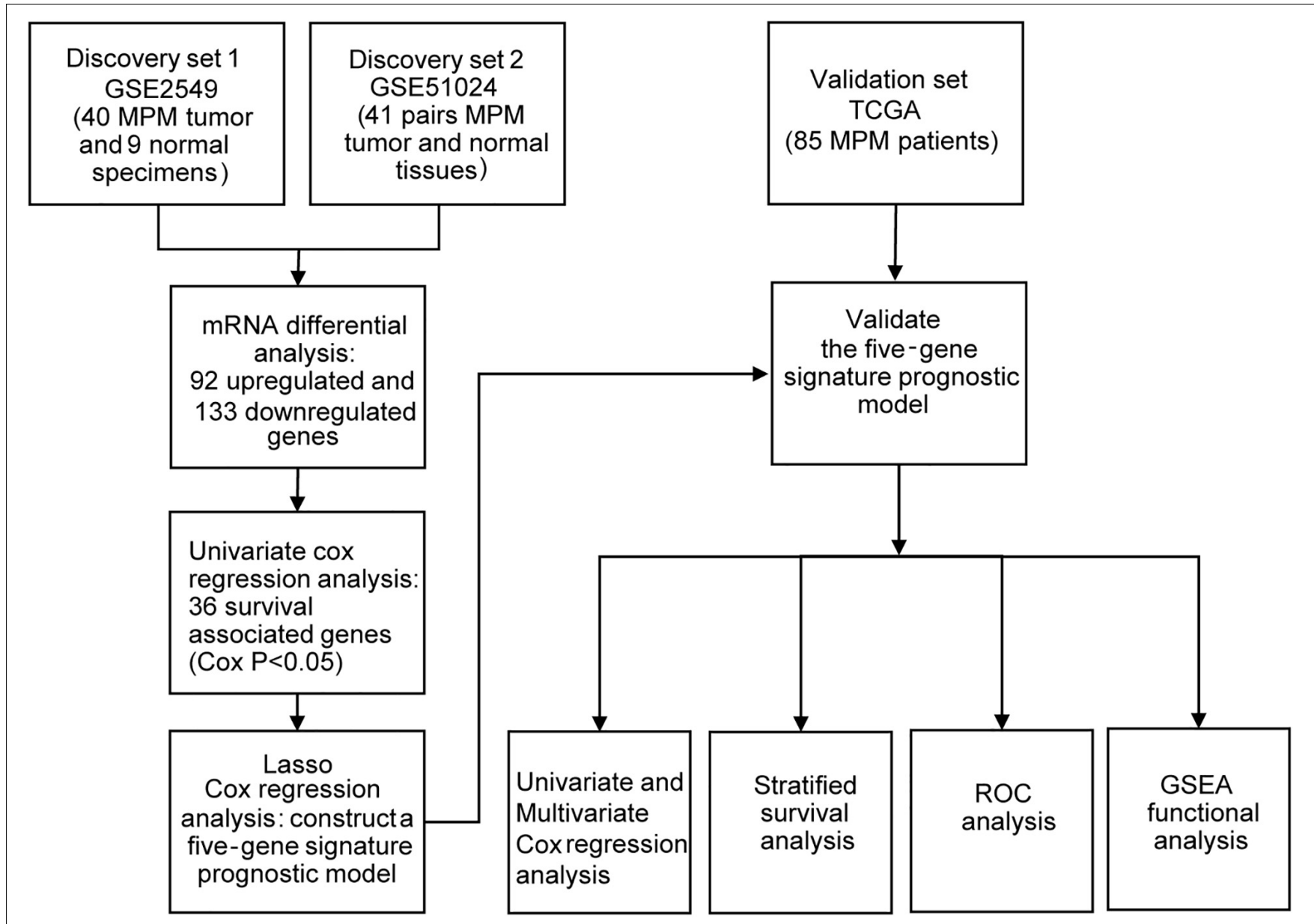

FIGURE 1 | Flowchart of the study design.

forward stepwise regression, bidirectional stepwise regression and relaxed LASSO, to construct the prognostic models and compare them with the five-gene signature. We found the genes selected by the three methods overlapped with the five-gene signature which confirmed the validity of the genes selected by LASSO Cox regression method (Supplementary Tables 1, 2 and Supplementary Figure 1). We then used AIC, C-index and ROC curves to evaluate and compare between the models and found the five-gene model showed overall better performance (Supplementary Tables 2, 3 and Supplementary Figure 2). We further validated the differential expressions of the five genes between tumor and normal tissues in GSE2549 and GSE51024 sets. All five genes were significantly overexpressed in tumor tissues (Supplementary Figure 3A). At the same time, we did the Kaplan-Meier curve to evaluate the relationship between the gene expression and the overall survival (OS). The five genes were strongly negatively related to the OS in GSE2549 dataset (Supplementary Figure 4).

Each patient's risk score was calculated according to the risk score model in the discovery cohort GSE2549. The patients were divided into low-risk or high-risk group by the median risk score. Patients in high-risk group had much shorter survival time than those in low-risk group (hazard ratio $=3.909,95 \%$ $\mathrm{CI}=1.797-8.503, \log$-rank test $P<0.0001)$. Chi-square analysis showed the death rate was significantly higher in high-risk group than low-risk group (Figures 3A-C). Simultaneously, we used MPM TCGA dataset ( 85 cases) as a validation cohort to confirm the reproducibility of the risk score model (Figures 3D-F). The prognostic signature was successfully validated in TCGA dataset showing that patients in high-risk group had significantly shorter OS than low-risk group patients (hazard ratio $=3.929,95 \%$ $\mathrm{CI}=2.295-6.726$, log-rank test $P<0.0001)$. In addition, all five genes were validated significantly negatively related to the OS in TCGA dataset (Supplementary Figure 5).

To evaluate whether the five-gene signature could be an independent prognostic factor for MPM, we conducted the univariate and multivariate Cox regression analyses in both GSE2549 and TCGA datasets. From the univariate analysis of GSE2549 dataset in Table 1, we observed that patients with mixed histological subtype, lymph node positive or high risk score were more likely to have shorter survival time and worse prognosis compared to patients with epithelial subtype, lymph 

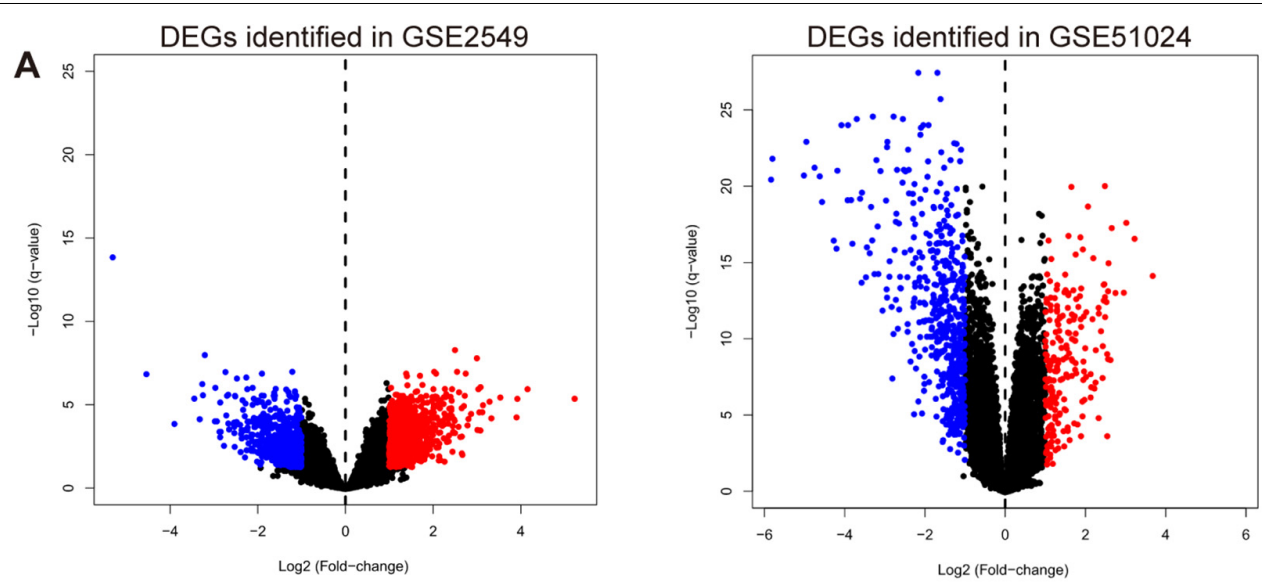

B Upregulated genes

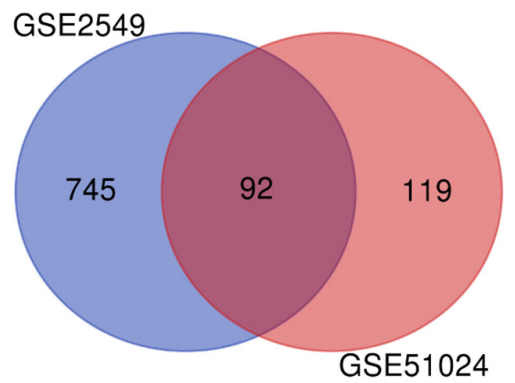

Downregulated genes

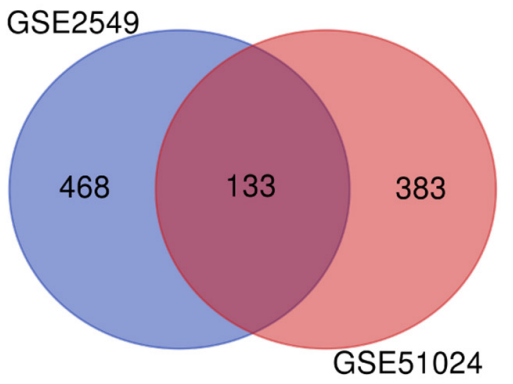

C
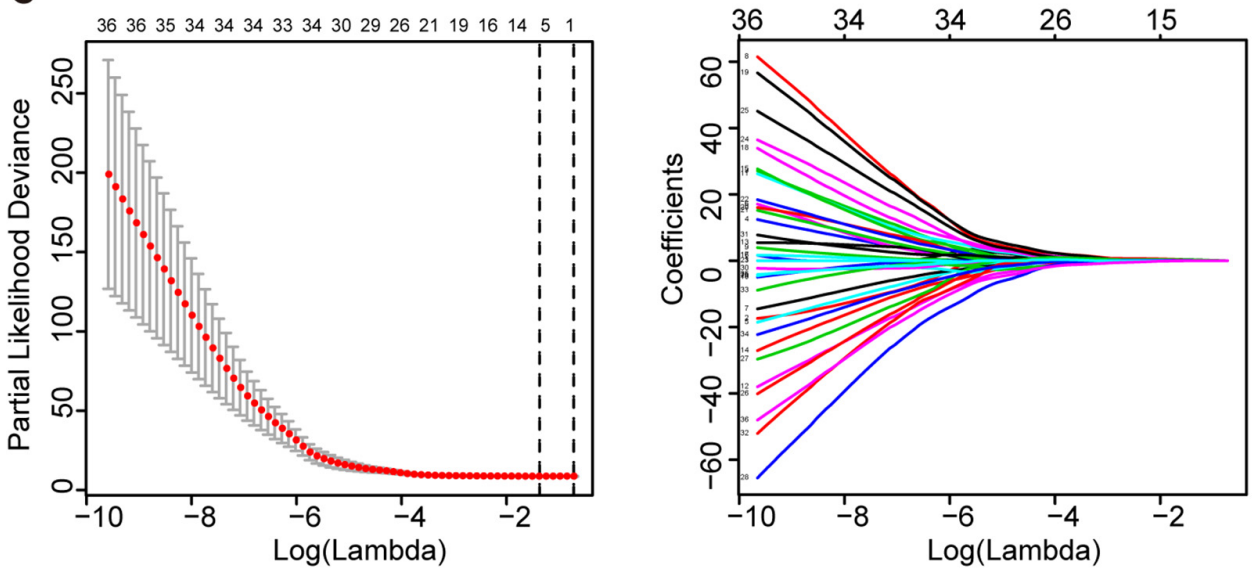

FIGURE 2 | Construction of the five-gene risk score model. (A) The volcano plots of GSE2549 and GSE51024 showing the DEGs between MPM tumor and normal tissues. $\mid \log _{2}$ Fold Change| $>1, q-<0.05$ for DEGs. Blue dot represents the significant downregulated genes and red dot represents the significant upregulated genes. (B) Venn of the DEGs in gene expression datasets GSE2549 and GSE51024. (C) Left panel: Plots of the cross-validation error rates. The two vertical dotted line represent the largest lambda value with minimum error (left) and 1- standard error (right); Right panel: LASSO coefficient profiles of the 36 genes detected by univariate cox regression analysis.

node negative or low-risk score. Hence, these results showed that histological subtype, node status and risk score had a relatively significant impact on prognosis of MPM patients. From the univariate analysis of TCGA dataset in Table 2, risk score was the only factor found to influence the prognosis of MPM patients. After controlling for gender, race, histological type, Brigham stage, AJCC stage, tumor size, lymphatic metastasis status and organ metastasis status, the multivariate analysis results showed the five-gene signature remained an independent prognostic factor in both datasets $(P<0.001$ in Table $1, P<0.0001$ in Table 2).

\section{Stratified Survival Analysis of the Five-Gene Risk Score Model}

Stratified survival analysis was further conducted in subgroups of patients with different clinical variables (gender, histological subtype, tumor size, lymph node metastasis status, AJCC stage, 

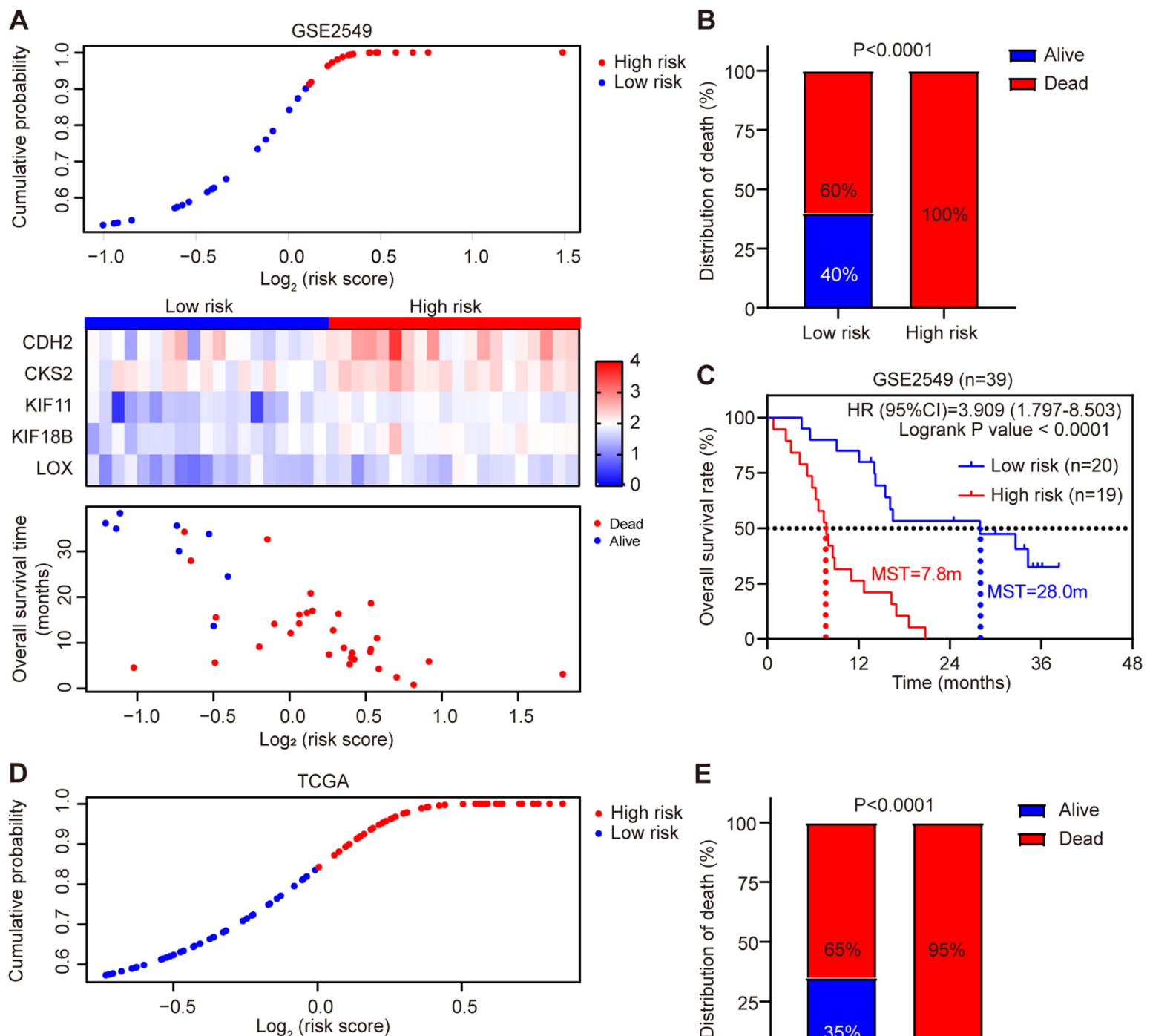

\section{E}
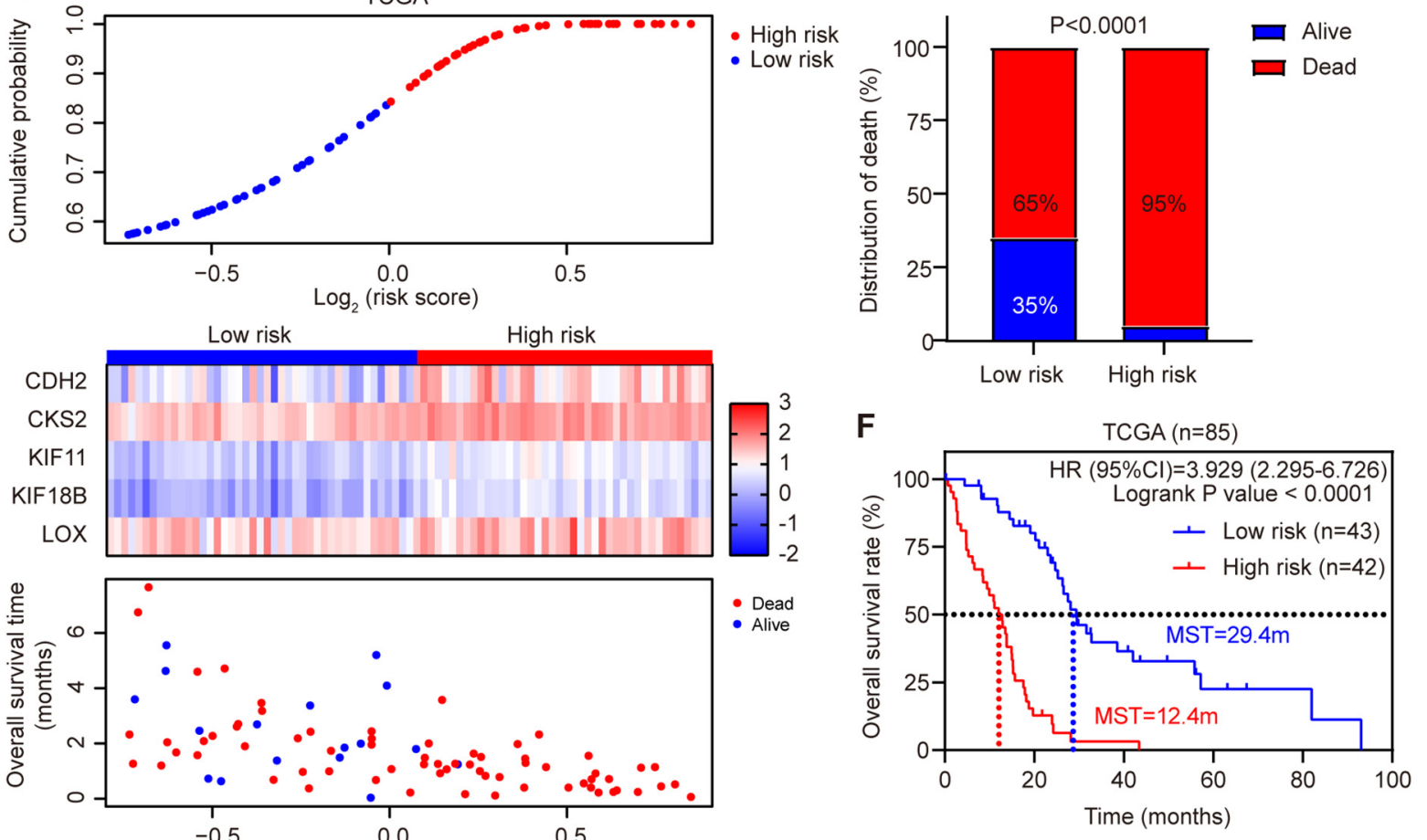

FIGURE 3 | Risk score distribution and validation of the five-gene signature risk score model in GSE2549 and TCGA datasets. (A,D) Upper panel: Low-risk and high-risk score distribution. Middle panel: the heatmap showing the five-gene expression profiles for each patient in low-risk and high-risk subgroups; Lower panel: the distribution of risk score with patient survival status. (B,E) Comparison of the MPM death rate in low-risk and high-risk groups. The death rate was higher in high-risk group than low-risk group. P-value was calculated by Chi-square test. (C,F) Kaplan-Meier survival analysis for the low-risk and high-risk patients (log-rank test). 
TABLE 1 | Univariate and multivariate survival analysis of GSE2549.

\begin{tabular}{|c|c|c|c|c|c|}
\hline \multirow[b]{2}{*}{ Variable } & \multicolumn{3}{|c|}{ Univariate analysis } & \multicolumn{2}{|c|}{ Multivariate analysis } \\
\hline & $\mathbf{N}$ & $P$-value & HR (95\% Cl) & $P$-value & HR (95\% Cl) \\
\hline \multicolumn{6}{|c|}{ Histological subtype } \\
\hline Epithelial & 23 & 0.038 & $2.232(1.047-4.759)$ & 0.717 & $0.834(0.312-2.226)$ \\
\hline Mixed & 16 & & & & \\
\hline \multicolumn{6}{|c|}{ Node status } \\
\hline NO & 8 & 0.045 & $2.945(1.025-8.461)$ & 0.089 & $3.060(0.843-11.106)$ \\
\hline$N+$ & 31 & & & & \\
\hline \multicolumn{6}{|l|}{ Margins } \\
\hline Negative & 5 & 0.347 & 1.771 (0.538-5.832) & 0.179 & $2.703(0.634-11.529)$ \\
\hline Positive & 34 & & & & \\
\hline \multicolumn{6}{|c|}{ Brigham stage } \\
\hline Stage $1+2$ & 12 & 0.394 & $1.404(0.644-3.064)$ & 0.628 & $0.784(0.292-2.101)$ \\
\hline Stage3 + 4 & 27 & & & & \\
\hline \multicolumn{6}{|l|}{ Risk score } \\
\hline Low-risk & 20 & $<0.0001$ & $4.963(2.190-11.247)$ & $<0.001$ & $5.995(2.178-16.502)$ \\
\hline High-risk & 19 & & & & \\
\hline
\end{tabular}

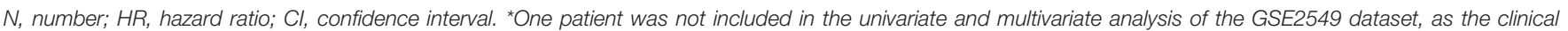
information was not available. All $P$ values in bold were less than 0.05 which were considered statistically significant.

and Brigham stage). According to Tables 3, 4, the five-gene risk score model was generally of statistically significant prognostic value. The MPM patients stratified by Brigham staging system, in either early stage or advanced stage, could be separated into the subgroups of better prognosis and poorer prognosis by the fivegene signature (Figures $4 \mathbf{A}, \mathbf{B}$ ). The model retained its prognostic value when stratified by AJCC staging system in TCGA dataset (Figures 4C,D). At the same time, we also found significant prognostic value of our model in lymph node negative or positive patients in both datasets (Figures 4E,F).

\section{Comparison of Five-Gene Risk Model With Other Clinicopathological Factors and Literature Models}

ROC analysis was conducted to evaluate the efficiency of our five-gene risk score model. The AUCs (area under curve) for the 1- and 2-years OS in GSE2549 dataset were $0.858(95 \%$ $\mathrm{CI}=0.727-0.990)$ and $0.959(95 \% \mathrm{CI}=0.918-1)($ Figures 5A,B $)$. We verified the efficiency using TCGA dataset. The AUCs for 1and 2-years OS were $0.821(95 \% \mathrm{CI}=0.726-0.916)$ and 0.852 (95\% CI $=0.766-0.938)$ (Figures 5C,D). Except for the 5-gene signature, we also did the ROC analyses of the clinicopathological factors available in either dataset (Supplementary Figures 6, 7). To further evaluate the prognostic value of the five-gene signature model, we compared our model with a three-gene prognostic model established by Zhou et al. (2019). The AUCs for 1- and 2years OS in either GSE2549 or TCGA dataset by our model were comparable with Zhou's model. We also used concordance index (C-index) to evaluate our model. And R package "compareC" was used to do the statistical comparison of the C-indices between our model and Zhou's model. The C-indices for GSE2549 dataset were comparable between our model and Zhou's model and no statistical difference was detected (Table 5). However, our model showed significant higher C-index value than Zhou's model $(P<0.05)$ in TCGA validation dataset (Table 6).

\section{Functional Analysis}

To obtain the potential biological function of the five-gene signature in MPM tumorigenesis, the Gene Set Enrichment Analysis (GSEA) was conducted to identify the associated pathways between the high-risk and low-risk subgroups in GSE2549 discovery dataset and TCGA validation dataset. Here we used the canonical pathway gene set from the Molecular Signatures database as our gene set database. The gene sets were considered significantly enriched when the definite value of normalized enrichment score was more than 1, Nominal $P$-value was less than 0.005 and FDR $q$-value was less than 0.05 . From the GSEA report, we discovered that "RHO GTPASES activate formins," "Mitotic spindle checkpoint," "PLK1 pathway," and "resolution of sister chromatid cohesion" pathways were significantly enriched in high-risk group patients from both discovery and validation sets. Simultaneously, several cancer related pathways such as "TP53 regulates transcription of cell cycle genes," "regulation of TP53 activity through phosphorylation," "cell cycle checkpoints," "kinesins," and "DNA double strand break repair" were also enriched in the high-risk group of TCGA. They were all reported to be involved in tumorigenesis and tumor progression (Supplementary Figure 8).

\section{DISCUSSION}

Over the years, several staging systems have been proposed for MPM (Rusch and Venkatraman, 1996). The TNM staging system proposed by the International Mesothelioma Interest Group (IMIG) subsequently accepted by the American Joint 
TABLE 2 | Univariate and multivariate survival analysis of TCGA.

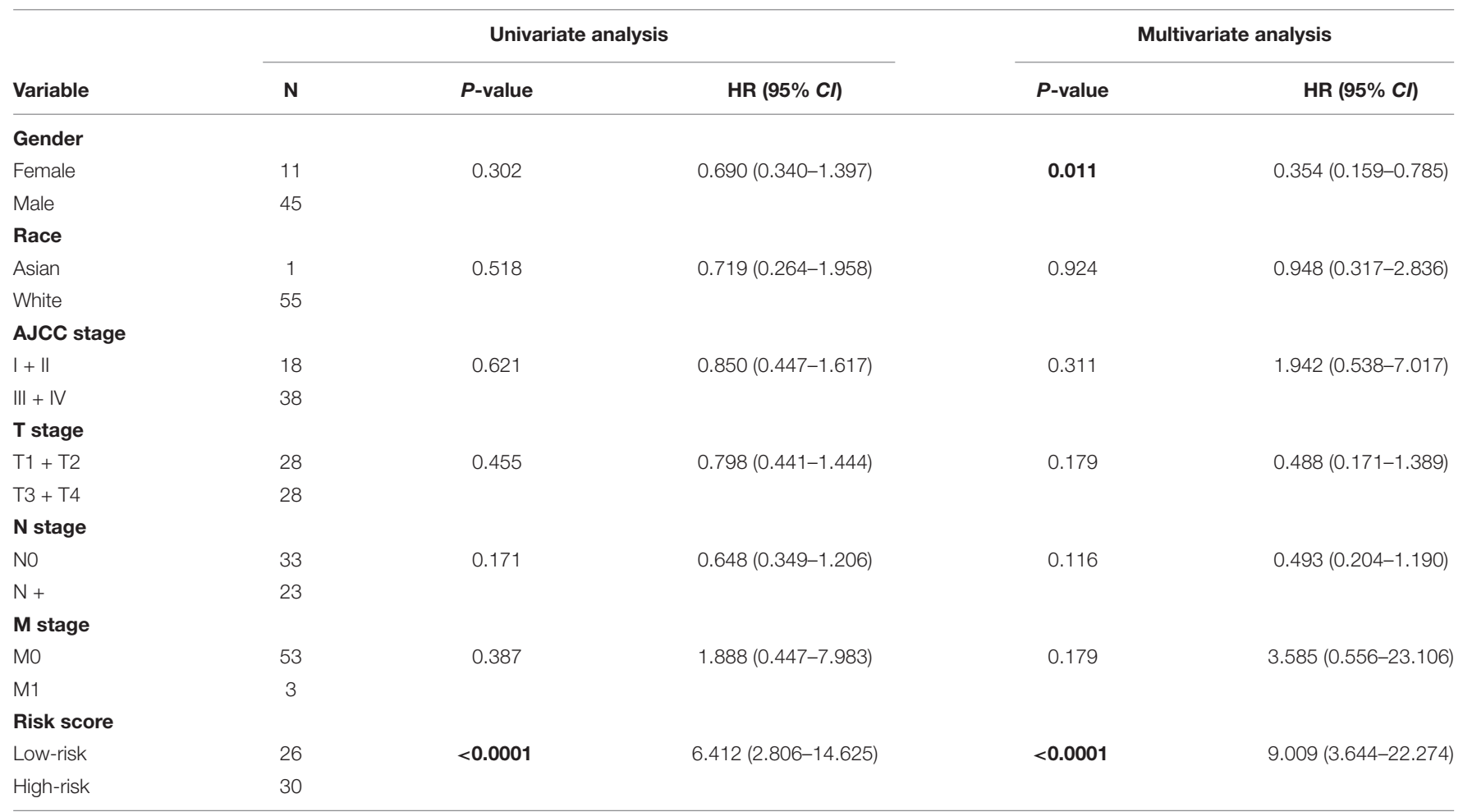

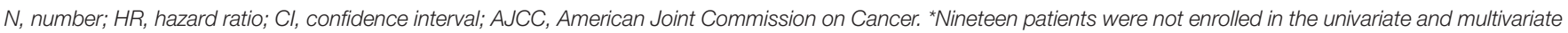
analysis of TCGA dataset, as some clinical information was not available. All $P$ values in bold were less than 0.05 which were considered statistically significant.

TABLE 3 | Stratified survival analysis of GSE2549.

\begin{tabular}{|c|c|c|c|c|}
\hline Variable & Low risk & High risk & $P$-value & HR (95\% Cl) \\
\hline \multicolumn{5}{|c|}{ Histological subtype } \\
\hline Epithelial & 15 & 8 & 0.0006 & $4.444(1.254-15.75)$ \\
\hline Mixed & 5 & 11 & 0.5396 & $1.390(0.4759-4.058)$ \\
\hline \multicolumn{5}{|l|}{ N stage } \\
\hline NO & 6 & 2 & 0.0473 & $5.486(0.3636-82.78)$ \\
\hline $\mathrm{N}+$ & 14 & 17 & 0.001 & $3.242(1.465-7.171)$ \\
\hline \multicolumn{5}{|c|}{ Brigham stage } \\
\hline$I+\|$ & 6 & 6 & 0.0138 & $4.529(1.098-18.69)$ \\
\hline $\mathrm{III}+\mathrm{IV}$ & 14 & 13 & 0.0012 & $3.485(1.391-8.727)$ \\
\hline
\end{tabular}

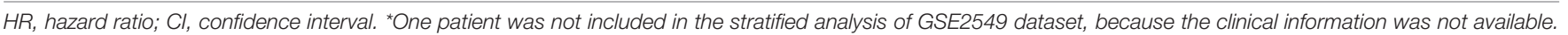
All $P$ values in bold were less than 0.05 which were considered statistically significant.

Commission on Cancer (AJCC) and the Union for International Cancer Control (UICC) is the one that has been generally accepted. Nevertheless, the current AJCC/UICC staging classification for MPM is still difficult to apply to clinical staging and thus may be imprecise in predicting prognosis and providing appropriate treatment for MPM patients (Rusch et al., 2012). Therefore, it is of great significance to explore biomarkers with optimal prognostic value for MPM patients. Research shows that combination of multiple biomarkers will improve the prognostic value instead of a single biomarker (Nalejska et al., 2014). In this study, we developed and validated a five-gene signature to evaluate the prognosis of MPM.
First, we identified 225 candidate DEGs based on two expression microarrays from GEO database. Aiming to eliminate the correlation between the genes selected by univariate analysis and improve the applicability in clinical practice, a five-gene signature risk score model was constructed by LASSO Cox regression. The LASSO Cox regression model has been widely applied to the Cox proportional hazard regression model for survival analysis with high dimensional data (Tibshirani, 1997; Zhang and Li, 2007; Wei et al., 2015; Lin et al., 2020). By studying Hastie et al's research work on comparing the LASSO with other model selection methods (Hastie et al., 2017), we introduced three more methods to confirm the validity of the 
TABLE 4 | Stratified survival analysis of TCGA.

\begin{tabular}{lcccc}
\hline Variable & Low-risk & High-risk & $\boldsymbol{P}$-value & HR (95\% Cl) \\
\hline $\begin{array}{l}\text { Gender } \\
\text { Female }\end{array}$ & 5 & 6 & $\mathbf{0 . 0 1 2 3}$ & $3.806(0.961-15.08)$ \\
Male & 21 & 24 & $\mathbf{0 . 0 0 0 1}$ & $3.110(1.559-6.204)$ \\
AJCC stage & & & & \\
I + II & 6 & 12 & $\mathbf{0 . 0 3 2 2}$ & $2.534(0.967-6.640)$ \\
III + IV & 20 & 18 & $\mathbf{0 . 0 0 0 6}$ & $3.174(1.444-6.973)$ \\
T stage & & & & \\
T1 + T2 & 12 & 16 & $\mathbf{0 . 0 0 1 8}$ & $2.889(1.268-6.582)$ \\
T3 + T4 & 14 & 14 & $\mathbf{0 . 0 0 2 5}$ & \\
N stage & & & & \\
No & 12 & 21 & $\mathbf{0 . 0 0 1 7}$ & $2.823(1.372-5.806)$ \\
N + & 14 & 9 & $\mathbf{0 . 0 0 1 3}$ & $3.937(1.163-13.33)$ \\
\hline
\end{tabular}

HR, hazard ratio; Cl, confidence interval; AJCC, American Joint Commission on Cancer. *Nineteen patients were not included in the stratified analysis of TCGA dataset, as some clinical information was not available. All $P$ values in bold were less than 0.05 which were considered statistically significant.

genes selected by LASSO Cox regression. The performances of the models were evaluated by AIC, C-index and AUC with respect to the degree of goodness-to-fit, the prediction accuracy and the predictive capacity. From the results, we could see the bidirectional stepwise regression showed the worst performance among these four methods (Supplementary Tables 2, 3 and Supplementary Figure 2). Our five-gene model by LASSO showed better or equivalent performance when compared with the models constructed by forward stepwise regression or relaxed LASSO. At the same time, we noticed four of the five genes (KIF18B, CKS2, LOX, and CDH2) in forward stepwise regression model and four of seven genes (CKS2, KIF11, KIF18B, and LOX) in relaxed LASSO model were overlapped with the five genes in LASSO model which further confirmed the validity of the genes selected by LASSO Cox regression method. Compared with the model constructed by relaxed LASSO method which included seven genes, our signature only had five genes and it showed better or equivalent prediction performance. The general principle for model selection was that for a given level of accuracy, a simpler or a more parsimonious model is preferable to a more complex one (Bozdogan, 1961; Stone, 1981). Taking into account the measurement cost to implement the model and the complexity of the model, the five- gene signature not only had better or equivalent prediction performance but also had higher practicability value.

The model was validated in GSE2549 and TCGA datasets showing that high-risk group patients always had worse clinical outcome and shorter survival time than low-risk group patients. In stratified analysis, our model had good performance in predicting OS. The univariate and multivariate analyses indicate our five-gene signature to be an independent prognostic factor for MPM patients. Simultaneously, our results showed that histological subtype, gender, and lymph node had relatively significant impact on prognosis, and these results were in agreement with many other researches for prognostic factors (Spirtas et al., 1988; Anand, 1994; Curran et al., 1998; Vigneswaran et al., 2017). To evaluate the accuracy and discrimination power of our risk score model, we did the ROC and C-index analyses. Simultaneously, we compared our model with available clinicopathological factors. Our risk score model exhibited stable predictive performance compared with clinicopathological factors (Supplementary Figures 6, 7). Considering our risk score model was using relatively objective gene expression levels tested by microarray or RNAseq while evaluating the prognosis of MPM patients, some of the clinicopathological factors might be variable in evaluation of prognosis of MPM patients. And this might also be due to lack of the data. We also compared our model with the literature model by Zhou et al. (2019), the AUCs for 1- and 2-years OS were comparable in discovery GSE2549 dataset. While putting the two models in the larger validation TCGA dataset, although our model showed higher AUCs, but the confidence of intervals overlapped, indicating no statistical difference. Therefore, we could not determine yet which model was better at evaluating the prognosis of MPM patients from the statistical perspective. Maybe due to the lack of data, we could not detect the statistical difference of AUCs between our model and Zhou's model. When we used C-index to compare our model and Zhou's model in the validation TCGA dataset, our model showed higher C-index value with a $P$-value of 0.000649 .

To gain more insights into the modulatory roles of the five genes in the signature, GSEA analysis was performed and showed that the "RHO GTPASES activate formins," "mitotic spindle checkpoint," "PLK1 pathway," and "resolution of sister chromatid cohesion" pathways were significantly associated with poor prognosis in high-risk subgroup MPM patients. The Rho family of GTPases is a family of small signaling $G$ protein that regulate actin cytoskeleton organization and dynamics (Vega and Ridley, 2008). Nearly 75\% of MPM cases harbor loss of function of core components of the Hippo pathway, which negatively regulates YAP activity (Zhang et al., 2010; Murakami et al., 2011). RhoA may strongly enhance YAP/TAZ activity, thereby promoting the proliferation of MPM in the sense that high YAP/TAZ activity is positively related to high proliferation capacity for MPM (Dupont et al., 2011; Mizuno et al., 2012). Zhang et al. reported a proliferation inhibitory effect in MPM cell lines with GSK269962A, a selective inhibitor of Rho-Kinase (Zhang et al., 2017). These studies, together with our result, indicated that the "Rho GTPASES activate formins" pathway is likely a significant mechanism in MPM tumorigenesis, and might serve as a target in the treatment of MPM patients. Additionally, the "mitotic spindle checkpoint" and "resolution of sister chromatid cohesion" pathways are closely related. The spindle checkpoint delays sister chromatid separation until all chromosomes have undergone bipolar spindle attachment. Dysfunction of this checkpoint contributes to tumorigenesis. Moreover, certain components of the mitotic spindle checkpoint pathway, including CHEK1, BUB1, and MAD2L1 were found to be upregulated in MPM tumors via microarray technology (Crispi et al., 2009). Similarly, Suraokar et al. carried out a gene expression microarray experiment on 53 surgically resected MPMs tumors along with paired normal tissues and found that the mitotic spindle checkpoint pathway was the most significantly altered pathway in MPM patients 

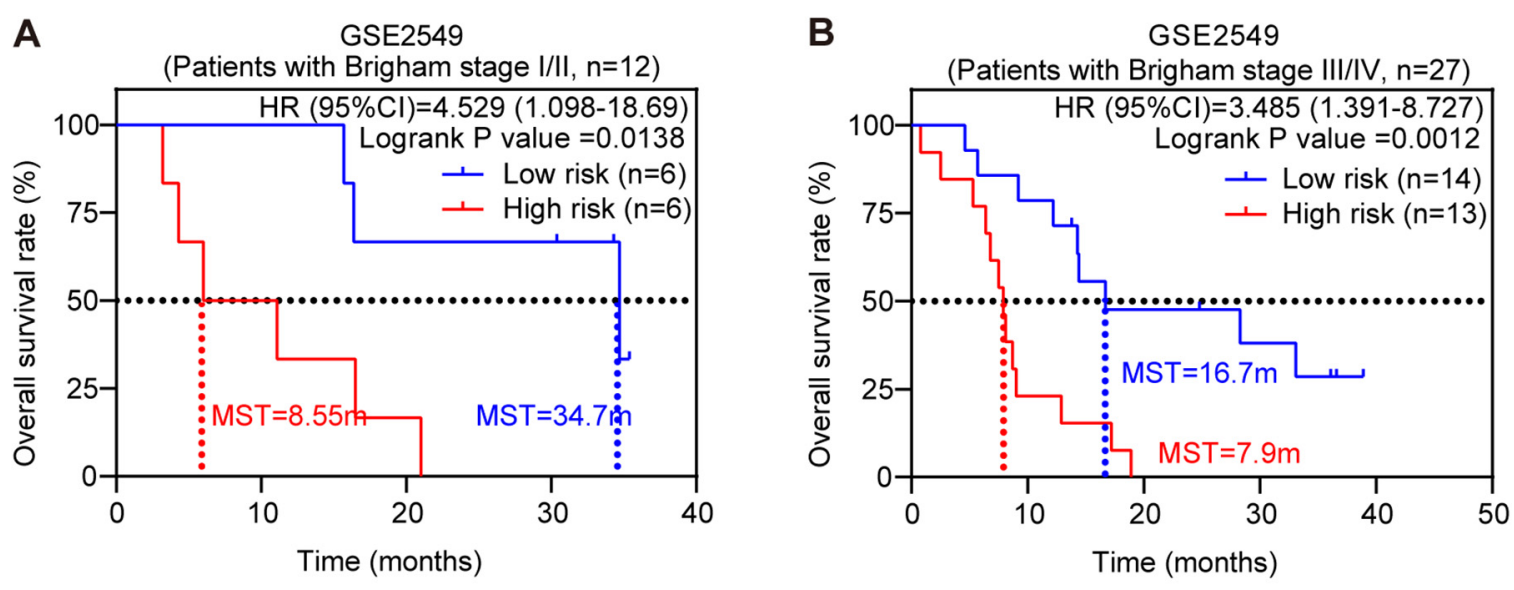

TCGA

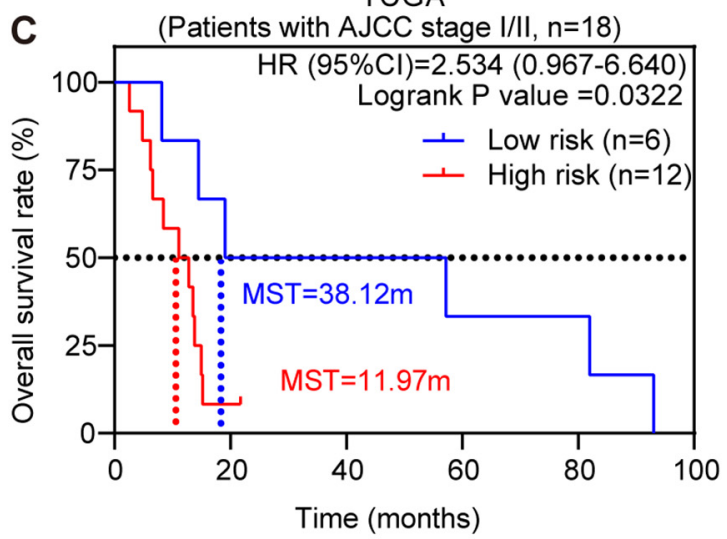

TCGA
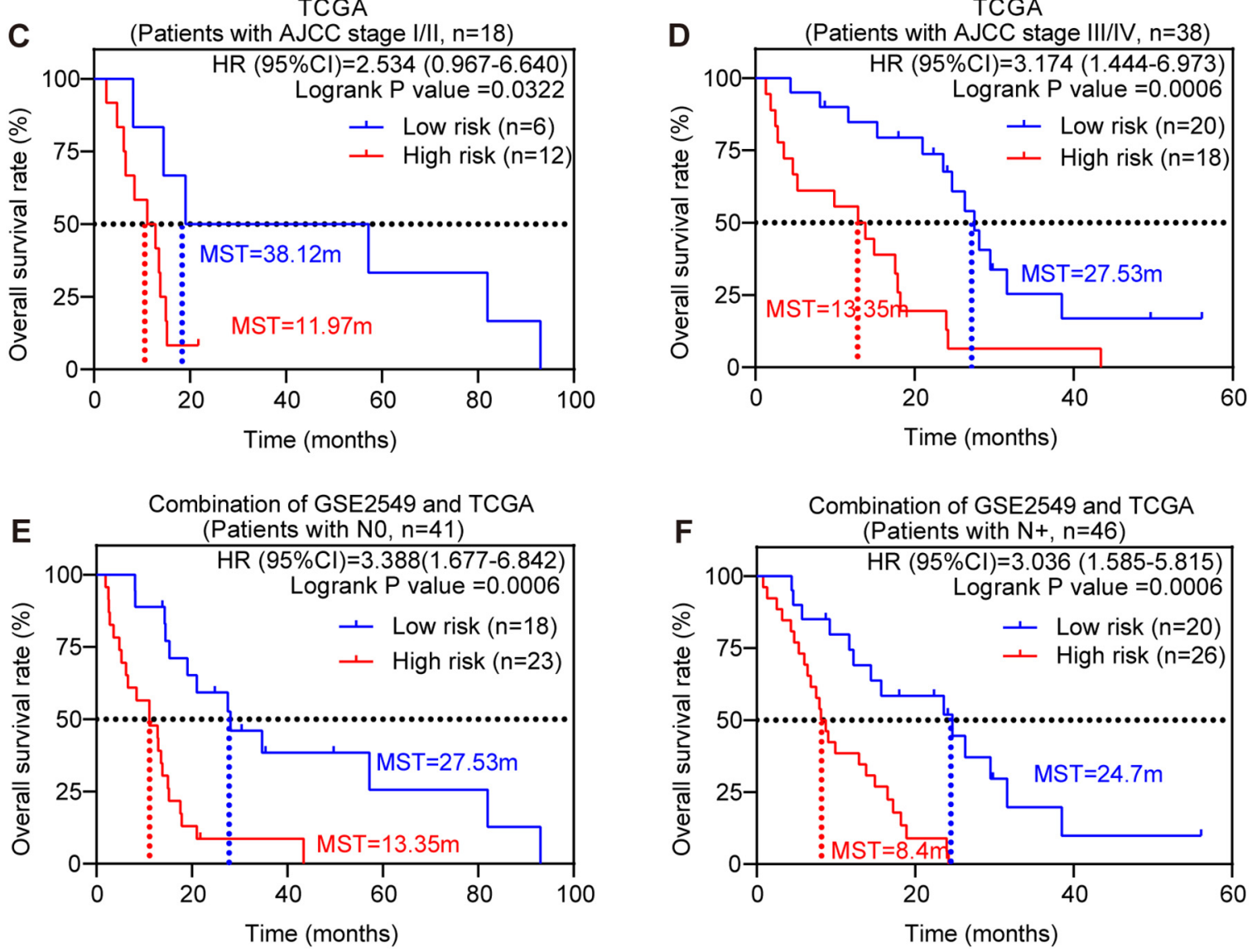

FIGURE 4 | Stratified survival analysis of the five-gene risk score model. Kaplan-Meier survival analysis for the patients with different clinical variables classified by the five-gene risk score model. (A) Patients in Brigham stage I/II; (B) patients in Brigham stage III/IV; (C) patients in AJCC stage I/II. (D) Patients in AJCC stage III/IV; (E) patients with lymph node negative; (F) patients with lymph node positive. P-values were calculated by Log-rank test.

(Suraokar et al., 2014b). They also evaluated the indicator for the deregulated expression of the mitotic spindle checkpoint pathway in an independent cohort of 80 MPM tumors and found higher nuclear MAD2L1 expression associated significantly $(P=0.043)$ with lower rates of OS. These findings, along with our result, clearly demonstrate the important role of the "mitotic spindle checkpoint" pathway in MPM that the pathway, suggesting that it might be a possible target for MPM therapy. Lastly, PLK1 (polo-like kinase 1), has been identified as a candidate therapeutic target and independent prognostic marker of MPM by an RNAi-based screening study (Linton et al., 2014). The inhibitory effect on cell proliferation following treatment with 


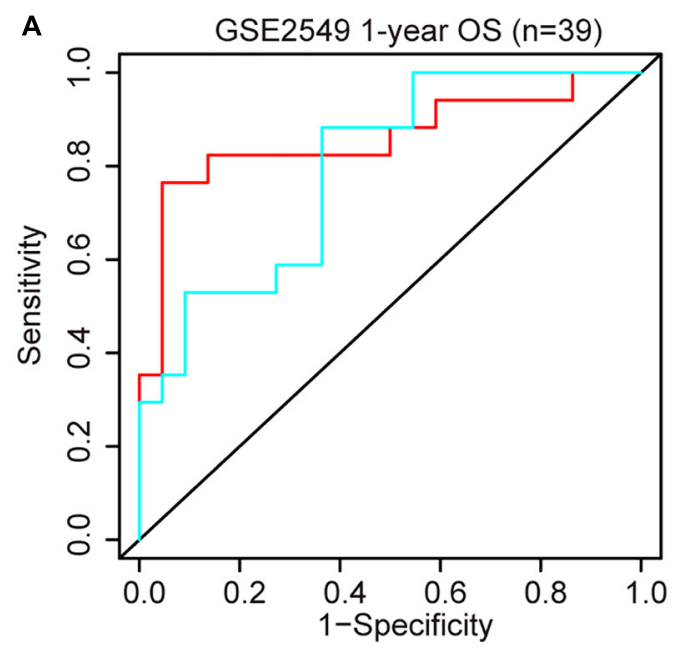

- Our Five-gene signature $\mathrm{AUC}=0.858$ $(95 \% \mathrm{Cl}=0.727-0.990)$

- Three-gene signature by Zhou et al AUC $=0.794$ $(95 \% \mathrm{Cl}=0.651-0.937)$

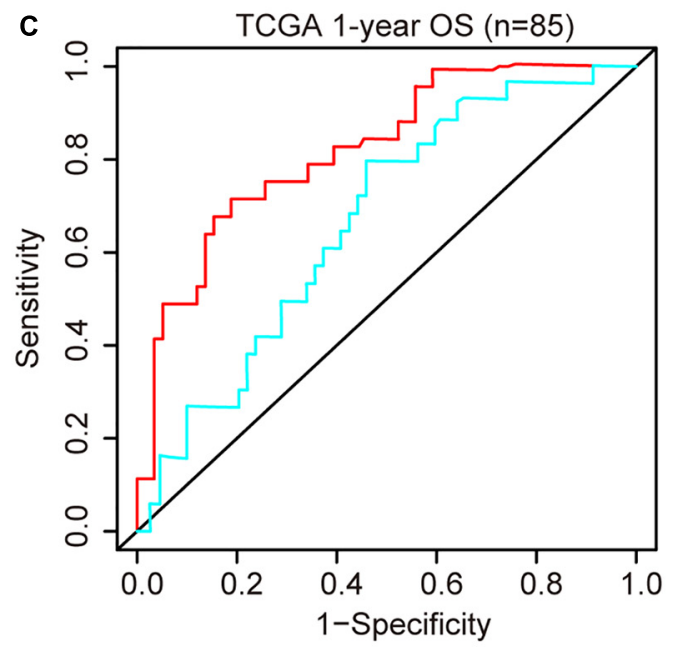

- Our Five-gene signature $A \cup C=0.821$ $(95 \% \mathrm{Cl}=0.726-0.916)$

- Three-gene signature by Zhou et al AUC $=0.666$ $(95 \% \mathrm{Cl}=0.545-0.788)$
B

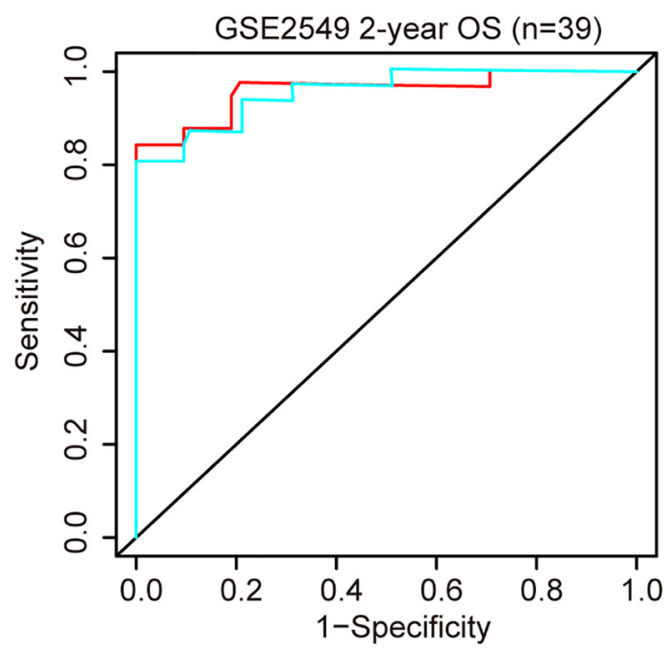

- Our Five-gene signature $A \cup C=0.959$ $(95 \% \mathrm{Cl}=0.918-1)$

- Three-gene signature by Zhou et al AUC $=0.956$ $(95 \% \mathrm{Cl}=0.905-1)$

D

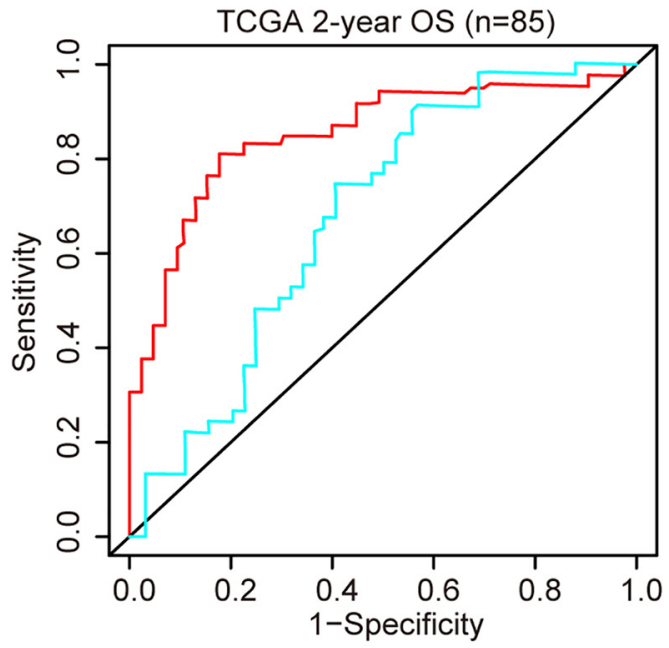

- Our Five-gene signature AUC $=0.852$ $(95 \% \mathrm{Cl}=0.766-0.938)$

- Three-gene signature by Zhou et al AUC $=0.679$ $(95 \% \mathrm{Cl}=0.56-0.799)$

FIGURE 5 | ROC curves of five-gene risk score model compared with the literature model by Zhou et al. (2019). The $X$ axis indicates false positive rate. The $Y$ axis indicates true positive rate. One patient in GSE2549 dataset was not enrolled in the analysis because the clinical information was not available. (A) 1-year OS in GSE2549; (B) 2-year OS in GSE2549; (C) 1-year OS in TCGA; (D) 2-year OS in TCGA.

the PLK1 small inhibitors BI6727, and BI2539 or the PLK1specific siRNA and artificial microRNA, have been validated in multiple MPM cell lines (Linton et al., 2014; Kato et al., 2016b). Hence, under our five-gene risk score classification, the high-risk MPM patients may benefit from PLK1 specific small molecule inhibitors, which are currently considered to be attractive therapeutic strategies against specific tumor types such as leukemia and non-small cell lung cancer (Medema et al., 2011; Lee et al., 2015).
All five genes (CDH2, CKS2, KIF11, KIF18B, and SEMA3G) in our model were confirmed to be significantly associated with the OS of MPM patients. Cadherin2 ( $\mathrm{CDH} 2)$, encoding N-cadherin protein, is closely related to the epithelial-mesenchymal transition (EMT) process and demonstrated prognostic significance in MPM (Schramm et al., 2010). It was reported that there was a substantial switch from epithelial markers such as E-cadherin and $\beta$-catenin to mesenchymal markers such as $\mathrm{N}$-cadherin through epithelium to biphasic and sarcomatoid 
TABLE 5 | C-index comparison in GSE2549 dataset.

\begin{tabular}{|c|c|c|c|}
\hline Signature & C-index & Z-score & $P$-value \\
\hline Our five-gene signature & $0.784(95 \% \mathrm{Cl}=0.674-0.894)$ & -0.35839 & 0.720051 \\
\hline Three-gene signature by Zhou et al. (2019) & $0.770(95 \% \mathrm{Cl}=0.725-0.815)$ & & \\
\hline
\end{tabular}

C-index, concordance index; $\mathrm{Cl}$, confidence interval.

TABLE 6 | C-index comparison in TCGA dataset.

\begin{tabular}{|c|c|c|c|}
\hline Signature & C-index & Z-score & $P$-value \\
\hline Our five-gene signature & $0.753(95 \% \mathrm{Cl}=0.698-0.808)$ & -3.41016 & 0.000649 \\
\hline Three-gene signature by Zhou et al. (2019) & $0.608(95 \% \mathrm{Cl}=0.567-0.649)$ & & \\
\hline
\end{tabular}

C-index, concordance index; $\mathrm{Cl}$, confidence interval. All P values in bold were less than 0.05 which were considered statistically significant.

subtypes, indicating the three histological subtypes of MPM are the consequences of different steps in an EMT process, of which the sarcomatoid subtype has the worst OS (Fassina et al., 2012). Consistent with these previous studies, we found the expression of $\mathrm{CDH} 2$ was higher in MPM tumor tissues than normal tissues and the high expression level of $\mathrm{CDH} 2$ was associated with poor prognosis (Supplementary Figures 3, 4A, 5A).

Cyclin-dependent kinases regulatory subunit 2 (CKS2) is the member of cell cycle dependent protein kinase subunits family. Although rarely reported with MPM, there is accumulating evidence showing CKS2 is upregulated in many types of tumor as a prognostic factor, including hepatocellular carcinoma, colorectal cancer, bladder cancer, breast cancer, gastric cancer and epithelium ovarian cancer (Kawakami et al., 2006; Shen et al., 2010; Tanaka et al., 2011; Yu et al., 2015; Huang et al., 2019; Xu et al., 2019). Our results revealed that the expression of CKS2 was elevated in MPM tumor tissues compared to normal tissues and it was harmful for the prognosis of MPM patients, which is in line with these previous findings of other types of tumor (Supplementary Figures 1, 4B, 5B). Investigators also found that CKS2 may advance tumor progression by promoting tumor cell proliferation and regulating apoptosis (Shen et al., 2013).

Kinesin family member 11 (KIF11, also known as EG5) and Kinesin family member $18 \mathrm{~B}$ (KIF18B) are two kinesin superfamily members, yet they are from two different kinesin subfamilies and function differently (Lawrence et al., 2004). KIF11 is essential for cell growth and proliferation, involved in the formation of the bipolar spindle in cell mitosis. KIF11 was found overexpressed not only in MPM human tumor samples and MPM human cell lines, but also in blast crisis chronic myelogenous leukemia and pancreatic cancer (Nowicki et al., 2003; Liu et al., 2010; Kato et al., 2016a). This is consistent with our analysis that KIF11was significantly overexpressed in MPM tumor tissues and was confirmed as a poor prognostic factor for MPM patients (Supplementary Figures 3, 4C, 5C). Mitotic arrest as a result of KIF11 inhibition has been observed in a variety of tumors (Infante et al., 2012). Several compounds inhibiting KIF11 entered Phase I and II clinical trials (ElNassan, 2013). Different from KIF11, KIF18B is involved in the regulation of microtubule dynamics (Lee et al., 2010). KIF18B was rarely reported with MPM, but it was reported promoting tumor progression in cervical cancer, hepatocellular carcinoma, and pancreatic cancer (Wu et al., 2018; Yang et al., 2020; Li et al., 2020). High levels of KIF18B were associated with poor prognosis in lung adenocarcinoma patients (Ji et al., 2019). Our analysis showed the high expression of KIF18B was significantly associated with poor prognosis of MPM patients (Supplementary Figures 4D, 5D).

Lysyl oxidase (LOX) was one of the five paralogs functioning primarily as the crosslink of collagens or elastin in extracellular matrix (Kim et al., 2011). Studies have shown that LOX mRNA level was increased in various cancer types, including head and neck squamous cell carcinoma, and breast and prostate cancers (Kirschmann et al., 2002; Lapointe et al., 2004; Erler et al., 2006). Recently, LOX was identified as a potential diagnostic biomarker in MPM (Kim et al., 2020). We also note that patients with high expression of LOX had shorter overall survival time and worse prognosis compared with patients with low expression of LOX (Supplementary Figures 4E, 5E).

Several limitations of our study should be pointed out. First, the clinical information of the patients was not comprehensive. Specifically, the clinical information of the discovery set GSE51024 was not accessible. In addition, there was no age, asbestos exposure history or any treatment information for the patients in either GSE2549 or TCGA dataset. Therefore, we could not evaluate additional possible prognostic factors. Second, given the low incidence of MPM, and the scarcity of available public databases, we could only adopt two datasets, GSE2549 and GSE51024, as our discovery datasets. GSE2549 dataset had a split of 40 tumor samples and 9 normal samples, while GSE51024 had 41 pairs of tumor and normal samples, this may lead to result bias and potentially cause the loss of viable DEGs. Third, only one MPM TCGA dataset was used as the validation dataset. The model was constructed based on the DEGs overlapping between the two discovery datasets using microarray technology, yet it was validated in the TCGA dataset using RNAseq technology which might affect the performance of the model. Compared with RNA-seq, microarray is unable to detect novel transcripts and might cause loss of possible DEGs, particularly genes with low expression. To test the practicality and accuracy of our model, rigorous validation in large prospective studies are needed. Finally, the molecular and biological mechanisms 
of these five genes in MPM need to be further investigated by additional research.

\section{CONCLUSION}

In conclusion, we have identified a five-gene signature risk score model as an objective and practical prognostic tool independent of clinicopathological factors for MPM patients, which complements the current MPM staging system. The accuracy and stability of our model provides opportunity for future clinical application.

\section{DATA AVAILABILITY STATEMENT}

Publicly available datasets were analyzed in this study. This data can be found here: https:/www.ncbi.nlm.nih.gov/geo/query/ acc.cgi?acc=GSE2549, https://www.ncbi.nlm.nih.gov/geo/query/ acc.cgi?acc=GSE51024, and https://www.cancer.gov/about-nci/ organization/ccg/research/structural-genomics/tcga.

\section{REFERENCES}

Anand, A. (1994). Prognostic factors of malignant mesothelioma of the pleura. Cancer 73:755. doi: 10.1002/1097-0142(19940201)73:3<755:: aid-cncr2820730343<3.0.co;2-u

Balatti, V., Maniero, S., Ferracin, M., Veronese, A., Negrini, M., Ferrocci, G., et al. (2011). MicroRNAs dysregulation in human malignant pleural mesothelioma. J. Thorac. Oncol. 6, 844-851. doi: 10.1097/JTO.0b013e31820db125

Benjamin, H., Lebanony, D., Rosenwald, S., Cohen, L., Gibori, H., Barabash, N., et al. (2010). A diagnostic assay based on microRNA expression accurately identifies malignant pleural mesothelioma. J. Mol. Diagn. 12, 771-779. doi: 10.2353/jmoldx.2010.090169

Bozdogan, H. (1961). Model selection and Akaike's information criterion (AIC): the general theory and its analytical extensions. Psychometrika, 52. 345-370. doi: 10.1007/BF02294361

Busacca, S., Germano, S., De Cecco, L., Rinaldi, M., Comoglio, F., Favero, F., et al. (2010). MicroRNA signature of malignant mesothelioma with potential diagnostic and prognostic implications. Am. J. Respir. Cell Mol. Biol. 42, 312-319. doi: 10.1165/rcmb.2009-0060OC

Crispi, S., Calogero, R. A., Santini, M., Mellone, P., Vincenzi, B., Citro, G., et al. (2009). Global gene expression profiling of human pleural mesotheliomas: identification of matrix metalloproteinase 14 (MMP-14) as potential tumour target. PLoS One. 4:e7016. doi: 10.1371/journal.pone.000 7016

Curran, D., Sahmoud, T., Therasse, P., van Meerbeeck, J., Postmus, P. E., and Giaccone, G. (1998). Prognostic factors in patients with pleural mesothelioma: the european organization for research and treatment of cancer experience. J. Clin. Oncol. 16, 145-152. doi: 10.1200/JCO.1998.16.1.145

Dupont, S., Morsut, L., Aragona, M., Enzo, E., Giulitti, S., Cordenonsi, M., et al. (2011). Role of YAP/TAZ in mechanotransduction. Nature 474, 179-183. doi: 10.1038 /nature 10137

El-Nassan, H. B. (2013). Advances in the discovery of kinesin spindle protein (Eg5) inhibitors as antitumor agents. Eur. J. Med. Chem. 62, 614-631. doi: 10.1016/j.ejmech.2013.01.031

Erler, J. T., Bennewith, K. L., Nicolau, M., Dornhofer, N., Kong, C., Le, Q. T., et al. (2006). Lysyl oxidase is essential for hypoxia-induced metastasis. Nature 440, 1222-1226. doi: 10.1038/nature04695

Fassina, A., Cappellesso, R., Guzzardo, V., Dalla Via, L., Piccolo, S., Ventura, L., et al. (2012). Epithelial-mesenchymal transition in malignant mesothelioma. Mod. Pathol. 25, 86-99. doi: 10.1038/modpathol.2011.144

\section{AUTHOR CONTRIBUTIONS}

$\mathrm{YB}, \mathrm{XW}, \mathrm{JH}, \mathrm{LG}, \mathrm{KN}$, and LJ contributed to the study design. $\mathrm{YB}, \mathrm{XW}, \mathrm{XL}, \mathrm{ZR}, \mathrm{LJ}$, and $\mathrm{KN}$ contributed to the statistical analysis. $\mathrm{YB}, \mathrm{ZR}$, and $\mathrm{HG}$ contributed to the manuscript draft. $\mathrm{YB}, \mathrm{XW}, \mathrm{LJ}$, and KN prepared the figures and tables. All authors reviewed and approved the final manuscript and carried out in collaboration work.

\section{ACKNOWLEDGMENTS}

We thank the Gene Expression Omnibus and the Cancer Genome Atlas offering convenient access to the MPM datasets.

\section{SUPPLEMENTARY MATERIAL}

The Supplementary Material for this article can be found online at: https://www.frontiersin.org/articles/10.3389/fgene. 2020.00899/full\#supplementary-material

Friedman, J., Hastie, T., Tibshirani, R., Narasimhan, B., Tay, K., Simon, N., Qian, J. (2020). Glmnet. Available online at: https://cran.r-project.org/web/packages/ glmnet/index.html (accessed February 1, 2020).

Gavin, G. J., Rockwell GN, Jensen RV, Rheinwald JG., Rheinwald, J. G., Glickman, J. N., et al. (2005). Malignant pleural mesothelioma. Available online at: https: //www.ncbi.nlm.nih.gov/geo/query/acc.cgi?acc=GSE2549 (accessed December 10, 2019).

Goeman, J. J. (2010). L1 penalized estimation in the Cox proportional hazards model. Biom. J. 52, 70-84. doi: 10.1002/bimj.20090 0028

Gordon, G. J., Jensen, R. V., Hsiao, L. L., Gullans, S. R., Blumenstock, J. E., Ramaswamy, S., et al. (2002). Translation of microarray data into clinically relevant cancer diagnostic tests using gene expression ratios in lung cancer and mesothelioma. Cancer. Res. 62, 4963-4967.

Gordon, G. J., Rockwell, G. N., Jensen, R. V., Rheinwald, J. G., Glickman, J. N., Aronson, J. P., et al. (2005). Identification of novel candidate oncogenes and tumor suppressors in malignant pleural mesothelioma using large-scale transcriptional profiling. Am. J. Pathol. 166, 1827-1840. doi: 10.1016/S00029440(10)62492-3

Gordon, S., Hu, Y., Ritchie, M., Silver, J., Wettenhall, J., McCarthy, D., et al. (2020). Limma. Available online at: http://bioinf.wehi.edu.au/limma (accessed January 3, 2020).

Gui, J., and Li, H. (2005). Penalized Cox regression analysis in the highdimensional and low-sample size settings, with applications to microarray gene expression data. Bioinformatics 21, 3001-3008. doi: 10.1093/bioinformatics/ bti422

Hastie, T. J., Tibshirani, R., Tibshirani, R. J. (2017). Extended Comparisons of Best Subset Selection, Forward Stepwise Selection, and the Lasso. arXiv [Preprint]. Available online at: https://arxiv.org/abs/1707.08692 (accessed May 17, 2020).

Heagerty, P. J., Lumley, T., and Pepe, M. S. (2000). Time-dependent ROC curves for censored survival data and a diagnostic marker. Biometrics 56, 337-344. doi: 10.1111/j.0006-341x.2000.00337.x

Herndon, J. E., Green, M. R., Chahinian, A. P., Corson, J. M., Suzuki, Y., and Vogelzang, N. J. (1998). Factors predictive of survival among 337 patients with mesothelioma treated between 1984 and 1994 by the Cancer and Leukemia Group B. Chest 113, 723-731. doi: 10.1378/chest.113.3.723

Hollevoet, K., Reitsma, J. B., Creaney, J., Grigoriu, B. D., Robinson, B. W., Scherpereel, A., et al. (2012). Serum mesothelin for diagnosing malignant pleural mesothelioma: an individual patient data meta-analysis. J. Clin. Oncol. 30, 1541-1549. doi: 10.1200/JCO.2011.39.6671 
Huang, N., Wu, Z., Hong, H., Wang, X., Yang, F., and Li, H. (2019). Overexpression of CKS2 is associated with a poor prognosis and promotes cell proliferation and invasion in breast cancer. Mol. Med. Rep. 19, 4761-4769. doi: 10.3892/mmr. 2019.10134

Infante, J. R., Kurzrock, R., Spratlin, J., Burris, H. A., Eckhardt, S. G., Li, J., et al. (2012). A Phase I study to assess the safety, tolerability, and pharmacokinetics of AZD4877, an intravenous Eg5 inhibitor in patients with advanced solid tumors. Cancer Chemother Pharmacol. 69, 165-172. doi: 10.1007/s00280-011-1667-z

Ji, Z., Pan, X., Shang, Y., Ni, D. T., and Wu, F. L. (2019). KIF18B as a regulator in microtubule movement accelerates tumor progression and triggers poor outcome in lung adenocarcinoma. Tissue Cell. 61, 44-50. doi: 10.1016/j.tice. 2019.09.001

Kang, L., Chen, W., Petrick, N. A., and Gallas, B. D. (2015). Comparing two correlated $\mathrm{C}$ indices with right-censored survival outcome: a one-shot nonparametric approach. Stat. Med. 34, 685-703. doi: 10.1002/sim.6370

Kassambara, A., Kosinski, M., Biecek, P., Fabian, S. (2020). CRAN - Package Survminer. Available online at: https://cran.r-project.org/web/packages/ survminer/index.html (accessed February 7, 2020).

Kato, T., Lee, D., Wu, L., Patel, P., Young, A. J., Wada, H., et al. (2016a). Kinesin family members KIF11 and KIF23 as potential therapeutic targets in malignant pleural mesothelioma. Int. J. Oncol. 49, 448-456. doi: 10.3892/ijo.2016.3566

Kato, T., Lee, D., Wu, L., Patel, P., Young, A. J., Wada, H., et al. (2016b). SORORIN and PLK1 as potential therapeutic targets in malignant pleural mesothelioma. Int. J. Oncol. 49, 2411-2420. doi: 10.3892/ijo.2016.3765

Kawakami, K., Enokida, H., Tachiwada, T., Gotanda, T., Tsuneyoshi, K., Kubo, H., et al. (2006). Identification of differentially expressed genes in human bladder cancer through genome-wide gene expression profiling. Oncol. Rep. 16, 521-531.

Kim, M. K., Kim, H. W., Jang, M., Oh, S. S., Yong, S. J., Jeong, Y., et al. (2020). LOX family and ZFPM2 as novel diagnostic biomarkers for malignant pleural mesothelioma. Biomark Res. 8:1. doi: 10.1186/s40364-019-0180-0

Kim, Y. M., Kim, E. C., and Kim, Y. (2011). The human lysyl oxidase-like 2 protein functions as an amine oxidase toward collagen and elastin. Mol. Biol. Rep. 38, 145-149. doi: 10.1007/s11033-010-0088-0

Kindler, H. L., Ismaila, N., Armato, S. G. III, Bueno, R., Hesdorffer, M., Jahan, T., et al. (2018). Treatment of malignant pleural mesothelioma: american society of clinical oncology clinical practice guideline. J. Clin. Oncol. 36, 1343-1373. doi: 10.1200/JCO.2017.76.6394

Kirschmann, D. A., Seftor, E. A., Fong, S. F., Nieva, D. R., Sullivan, C. M., Edwards, E. M., et al. (2002). A molecular role for lysyl oxidase in breast cancer invasion. Cancer Res. 62, 4478-4483.

Kirschner, M. B., Cheng, Y. Y., Armstrong, N. J., Lin, R. C., Kao, S. C., Linton, A., et al. (2015). MiR-score: a novel 6-microRNA signature that predicts survival outcomes in patients with malignant pleural mesothelioma. Mol. Oncol. 9, 715-726. doi: 10.1016/j.molonc.2014.11.007

Lapointe, J., Li, C., Higgins, J. P., van de Rijn, M., Bair, E., Montgomery, K., et al. (2004). Gene expression profiling identifies clinically relevant subtypes of prostate cancer. Proc. Natl. Acad. Sci. U.S.A. 101, 811-816. doi: 10.1073/pnas. 0304146101

Lawrence, C. J., Dawe, R. K., Christie, K. R., Cleveland, D. W., Dawson, S. C., Endow, S. A., et al. (2004). A standardized kinesin nomenclature. J. Cell Biol. 167, 19-22. doi: $10.1083 /$ jcb. 200408113

Le Kang, W. C. (2015). compareC: Compare Two Correlated C Indices with Rightcensored Survival Outcome. Available online at: https://rdrr.io/cran/compareC/ (accessed July 1, 2020).

Lee, K. S., Burke, T. R. Jr., Park, J. E., Bang, J. K., and Lee, E. (2015). recent advances and new strategies in targeting Plk1 for anticancer therapy. Trends Pharmacol. Sci. 36, 858-877. doi: 10.1016/j.tips.2015.08.013

Lee, Y. M., Kim, E., Park, M., Moon, E., Ahn, S. M., Kim, W., et al. (2010). Cell cycle-regulated expression and subcellular localization of a kinesin- 8 member human KIF18B. Gene 466, 16-25. doi: 10.1016/j.gene.2010.06.007

Li, B., Liu, B., Zhang, X., Liu, H., and He, L. (2020). KIF18B promotes the proliferation of pancreatic ductal adenocarcinoma via activating the expression of CDCA8. J. Cell Physiol. 235, 4227-4238. doi: 10.1002/jcp.29201

Liberzon, A., Subramanian, A., Pinchback, R., Thorvaldsdottir, H., Tamayo, P., and Mesirov, J. P. (2011). Molecular signatures database (MSigDB) 3.0. Bioinformatics 27, 1739-1740. doi: 10.1093/bioinformatics/btr260
Lin, R. T., Chien, L. C., Jimba, M., Furuya, S., and Takahashi, K. (2019). Implementation of national policies for a total asbestos ban: a global comparison. Lancet Planet Health 3, e341-e348. doi: 10.1016/S2542-5196(19) 30109-3

Lin, Y., Pan, X., Chen, Z., Lin, S., and Chen, S. (2020). Identification of an immunerelated nine-lncrna signature predictive of overall survival in colon cancer. Front Genet. 11:318. doi: 10.3389/fgene.2020.00318

Linton, A., Cheng, Y. Y., Griggs, K., Schedlich, L., Kirschner, M. B., Gattani, S., et al. (2014). An RNAi-based screen reveals PLK1, CDK1 and NDC80 as potential therapeutic targets in malignant pleural mesothelioma. Br. J. Cancer. 110, 510-519. doi: 10.1038/bjc.2013.731

Liu, M., Wang, X., Yang, Y., Li, D., Ren, H., Zhu, Q., et al. (2010). Ectopic expression of the microtubule-dependent motor protein Eg5 promotes pancreatic tumourigenesis. J. Pathol. 221, 221-228. doi: 10.1002/path.2706

Mazurek, J. M. M. D., Syamlal, G., Wood, J. M., Hendricks, S. A., and Weston, A. (2017). Malignant Mesothelioma Mortality - United States, 1999-2015. MMWR Morbidity and Mortality Weekly Report2017. Available online at: https://www.cdc.gov/mmwr/volumes/66/wr/mm6608a3.htm (accessed March 7, 2020).

Medema, R. H., Lin, C. C., and Yang, J. C. (2011). Polo-like kinase 1 inhibitors and their potential role in anticancer therapy, with a focus on NSCLC. Clin. Cancer Res. 17, 6459-6466. doi: 10.1158/1078-0432.CCR-11-0541

Mizuno, T., Murakami, H., Fujii, M., Ishiguro, F., Tanaka, I., Kondo, Y., et al. (2012). YAP induces malignant mesothelioma cell proliferation by upregulating transcription of cell cycle-promoting genes. Oncogene 31, 5117-5122. doi: 10. 1038/onc. 2012.5

Murakami, H., Mizuno, T., Taniguchi, T., Fujii, M., Ishiguro, F., Fukui, T., et al. (2011). LATS2 is a tumor suppressor gene of malignant mesothelioma. Cancer Res. 71, 873-883. doi: 10.1158/0008-5472.CAN-10-2164

My.stepwise.coxph (2017). Stepwise Variable Selection Procedure for Cox's Proportional Hazards Model and Cox's Model. Available online at: https: //rdrr.io/cran/My.stepwise/man/My.stepwise.coxph.html (accessed June 1, 2020).

Nalejska, E., Maczynska, E., and Lewandowska, M. A. (2014). Prognostic and predictive biomarkers: tools in personalized oncology. Mol. Diagn. Ther. 18, 273-284. doi: 10.1007/s40291-013-0077-9

Nowicki, M. O., Pawlowski, P., Fischer, T., Hess, G., Pawlowski, T., and Skorski, T. (2003). Chronic myelogenous leukemia molecular signature. Oncogene 22, 3952-3963. doi: 10.1038/sj.onc. 1206620

Patrick (2013). Heagerty pbPS-C. survivalROC: Time-Dependent ROC Curve Estimation from Censored Survival Data. Available online at: https://cran. r-project.org/web/packages/survivalROC/index.html (accessed December 30, 2019).

Ritchie, M. E., Phipson, B., Wu, D., Hu, Y., Law, C. W., Shi, W., et al. (2015). limma powers differential expression analyses for RNA-sequencing and microarray studies. Nucleic Acids Res. 43:e47. doi: 10.1093/nar/gkv007

Rusch, V. W. (1995). A proposed new international TNM staging system for malignant pleural mesothelioma. From the international mesothelioma interest group. Chest 108, 1122-1128. doi: 10.1378/chest.108.4.1122

Rusch, V. W., Giroux, D., Kennedy, C., Ruffini, E., Cangir, A. K., Rice, D., et al. (2012). Initial analysis of the international association for the study of lung cancer mesothelioma database. J. Thorac. Oncol. 7, 1631-1639. doi: 10.1097/ JTO.0b013e31826915f1

Rusch, V. W., and Venkatraman, E. (1996). The importance of surgical staging in the treatment of malignant pleural mesothelioma. J. Thorac. Cardiovasc. Surg 111, 815-825. doi: 10.1016/s0022-5223(96)70342-2

Schramm, A., Opitz, I., Thies, S., Seifert, B., Moch, H., Weder, W., et al. (2010). Prognostic significance of epithelial-mesenchymal transition in malignant pleural mesothelioma. Eur. J. Cardiothorac. Surg. 37, 566-572. doi: 10.1016/j. ejcts.2009.08.027

Shen, D. Y., Fang, Z. X., You, P., Liu, P. G., Wang, F., Huang, C. L., et al. (2010). Clinical significance and expression of cyclin kinase subunits 1 and 2 in hepatocellular carcinoma. Liver Int. 30, 119-125. doi: 10.1111/j.1478-3231. 2009.02106.x

Shen, D. Y., Zhan, Y. H., Wang, Q. M., Rui, G., and Zhang, Z. M. (2013). Oncogenic potential of cyclin kinase subunit-2 in cholangiocarcinoma. Liver Int. 33, 137-148. doi: 10.1111/liv.12014 
Spirtas, R., Connelly, R. R., and Tucker, M. A. (1988). Survival patterns for malignant mesothelioma: the SEER experience. Int J Cancer. 41, 525-530. doi: 10.1002/ijc. 2910410409

Stone, C. J. (1981). Admissible selection of an accurate and parsimonious normal linear regression model. Ann. Stat. 9, 475-485. doi: 10.1214/aos/1176345452

Suraokar, M. B., Nunez, M. I, Diao, L., and Chow, C. W. (2014a). Gene Expression of Malignant Pleural Mesothelioma Tumor and paired Normal Lung tissue. Available online at: https://www.ncbi.nlm.nih.gov/geo/query/acc.cgi (accessed December 14, 2019).

Suraokar, M. B., Nunez, M. I., Diao, L., Chow, C. W., Kim, D., Behrens, C., et al. (2014b). Expression profiling stratifies mesothelioma tumors and signifies deregulation of spindle checkpoint pathway and microtubule network with therapeutic implications. Ann. Oncol. 25, 1184-1192. doi: 10.1093/annonc/ mdu 127

Tanaka, F., Matsuzaki, S., Mimori, K., Kita, Y., Inoue, H., and Mori, M. (2011). Clinicopathological and biological significance of CDC28 protein kinase regulatory subunit 2 overexpression in human gastric cancer. Int J Oncol. 39, 361-372. doi: 10.3892/ijo.2011.1056

Terry, M., and Therneau, T. L. (2020). Atkinson Elizabeth, Crowson Cynthia survival: Survival Analysis. Available online at: https://cran.r-project.org/web/ packages/survival/index.html (accessed February 13, 2020).

Tibshirani, R. (1997). The lasso method for variable selection in the Cox model. Stat. Med. 16, 385-395. doi: 10.1002/(sici)1097-0258(19970228)16:4<385::aid$\operatorname{sim} 380<3.0 . c 0$

Vega, F. M., and Ridley, A. J. (2008). Rho GTPases in cancer cell biology. FEBS Lett. 582, 2093-2101. doi: 10.1016/j.febslet.2008.04.039

Vigneswaran, W. T., Kircheva, D. Y., Ananthanarayanan, V., Watson, S., Arif, Q., Celauro, A. D., et al. (2017). Amount of epithelioid differentiation is a predictor of survival in malignant pleural mesothelioma. Ann. Thorac. Surg. 103, 962-966. doi: 10.1016/j.athoracsur.2016.08.063

Vogelzang, N. J., Rusthoven, J. J., Symanowski, J., Denham, C., Kaukel, E., Ruffie, P., et al. (2003). Phase III study of pemetrexed in combination with cisplatin versus cisplatin alone in patients with malignant pleural mesothelioma. J. Clin. Oncol. 21, 2636-2644. doi: 10.1200/JCO.2003.11.136

Wagner, J. C., Sleggs, C. A., and Marchand, P. (1960). Diffuse pleural mesothelioma and asbestos exposure in the North Western Cape Province. Br. J. Ind. Med. 17, 260-271. doi: 10.1136/oem.17.4.260

Walker, A. M., Loughlin, J. E., Friedlander, E. R., Rothman, K. J., and Dreyer, N. A. (1983). Projections of asbestos-related disease 1980-2009. J. Occup. Med. 25, 409-425.

Wei, J. H., Haddad, A., Wu, K. J., Zhao, H. W., Kapur, P., Zhang, Z. L., et al. (2015). A CpG-methylation-based assay to predict survival in clear cell renal cell carcinoma. Nat. Commun. 6:8699. doi: 10.1038/ncomms9699
Wu, Y., Wang, A., Zhu, B., Huang, J., Lu, E., Xu, H., et al. (2018). KIF18B promotes tumor progression through activating the $\mathrm{Wnt} /$ beta-catenin pathway in cervical cancer. Onco. Targets Ther. 11, 1707-1720. doi: 10.2147/OTT.S157440

$\mathrm{Xu}$, J. H., Wang, Y., and Xu, D. (2019). CKS2 promotes tumor progression and metastasis and is an independent predictor of poor prognosis in epithelial ovarian cancer. Eur. Rev. Med. Pharmacol. Sci. 23, 3225-3234. doi: 10.26355/ eurrev_201904_17681

Yang, B., Wang, S., Xie, H., Wang, C., Gao, X., Rong, Y., et al. (2020). KIF18B promotes hepatocellular carcinoma progression through activating Wnt/betacatenin-signaling pathway. J. Cell Physiol. doi: 10.1002/jcp.29444

Yang, Y., and Zou, H. (2013a). A cocktail algorithm for solving the elastic net penalized cox's regression in high dimensions. Stat. Interface 6, 167-173. doi: 10.4310/SII.2013.v6.n2.a1

Yang, Y. and Zou, H. (2013b). fastcox: Lasso and Elastic-Net Penalized Cox's Regression in High Dimensions Models using the Cocktail Algorithm. Available online at: https://rdrr.io/cran/fastcox/ (accessed June 1, 2020).

Yu, M. H., Luo, Y., Qin, S. L., Wang, Z. S., Mu, Y. F., and Zhong, M. (2015). Upregulated CKS2 promotes tumor progression and predicts a poor prognosis in human colorectal cancer. Am. J. Cancer Res. 5, 2708-2718.

Zhang, H. H., and Li, W. (2007). Adaptive lasso for Cox's proportional hazards model Biometrika. 94, 691-703. doi: 10.1093/biomet/asm037

Zhang, N., Bai, H., David, K. K., Dong, J., Zheng, Y., Cai, J., et al. (2010). The Merlin/NF2 tumor suppressor functions through the YAP oncoprotein to regulate tissue homeostasis in mammals. Dev. Cell. 19, 27-38. doi: 10.1016/j. devcel.2010.06.015

Zhang, W. Q., Dai, Y. Y., Hsu, P. C., Wang, H., Cheng, L., Yang, Y. L., et al. (2017). Targeting YAP in malignant pleural mesothelioma. J. Cell Mol. Med. 21, 2663-2676. doi: 10.1111/jcmm.13182

Zhou, J. G., Zhong, H., Zhang, J., Jin, S. H., Roudi, R., and Ma, H. (2019). Development and validation of a prognostic signature for malignant pleural mesothelioma. Front. Oncol. 9:78. doi: 10.3389/fonc.2019.00078

Conflict of Interest: The authors declare that the research was conducted in the absence of any commercial or financial relationships that could be construed as a potential conflict of interest.

Copyright (C) 2020 Bai, Wang, Hou, Geng, Liang, Ruan, Guo, Nan and Jiang. This is an open-access article distributed under the terms of the Creative Commons Attribution License (CC BY). The use, distribution or reproduction in other forums is permitted, provided the original author(s) and the copyright owner(s) are credited and that the original publication in this journal is cited, in accordance with accepted academic practice. No use, distribution or reproduction is permitted which does not comply with these terms. 